International Journal of Current Advanced Research

ISSN: O: 2319-6475, ISSN: P: 2319 - 6505, Impact Factor: SJIF: 5.995

Available Online at www.journalijcar.org

Volume 6; Issue 5; May 2017; Page No. 3943-3947

DOI: http://dx.doi.org/10.24327/ijcar.2017.3947.0402

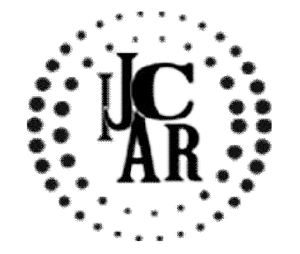

Research Article

\title{
A CLINICAL PROFILE OF POSTERIOR UVEITIS IN A MULTISPECIALTY HOSPITAL
}

\section{Radha Annamalai1., Samuel Cornelius Gnanadurai J² and Muthayya Muthukumar3}

1,30phthalmology, Sri Ramachandra University, Porur

${ }^{1}$ Ophthalmology Sri Muthukumaran Medical College, Mangadu, Chennai

\section{A R T I C L L $\mathbf{E} \quad$ I N F O}

\section{Article History:}

Received $5^{\text {th }}$ February, 2017

Received in revised form $10^{\text {th }}$ March, 2017

Accepted $8^{\text {th }}$ April, 2017

Published online $28^{\text {th }}$ May, 2017

\section{Key words:}

Posterior Uveitis, Tuberculosis, Choroiditis, Toxoplasma Retinochoroiditis, Serpiginous Choroiditis

\begin{abstract}
A B S T R A C T
Posterior uveitis and its sequelae are known to be sight threatening and has diverse etiologies and presentations. Identification of etiology is important as management is different in infections and autoimmune diseases. The aim of this study was to do a prospective analysis of 75 patients with posterior uveitis to identify frequency of etiology, most common presentation, complications and the cause of visual loss. A review of the history, clinical examination, ancillary investigations and laboratory tests were done for all patients at presentation and follow up. Infections were noted in $37 \%$ and autoimmune diseases in $33 \%$. The most frequent presentation was choroiditis. The most common etiology was tubercular posterior uveitis, toxoplasma and autoimmune serpiginous choroiditis. Recurrences and complications were observed.Posterior uveitis in our setting was observed in the $4^{\text {th }}$ decade of life and was mostly due to infection. Infections may present with posterior uveitis before becoming apparent assystemic disease.Macular complications weremost commonly cystoid macular edema and macular scarring.
\end{abstract}

Copyright $\mathrm{C} 2017$ Radha Annamalai et al. This is an open access article distributed under the Creative Commons Attribution License, which permits unrestricted use, distribution, and reproduction in any medium, provided the original work is properly cited.

\section{INTRODUCTION}

Posterior uveitis (PU) is a frequently encountered type of inflammation with speculations regarding its etiology, progress and its prognosis. In several clinical scenarios what may be perceived as due to asystemic infection ${ }^{1}$ may not actually be so and the underlying etiology may be an autoimmune process. It is of paramount importance to identify the possible etiology as PU can be infective or noninfective and treatment is entirely different in both. Besides, the multitude of presentations in PUin the form of choroiditis, retinochoroiditis, vasculitis and vitritis ${ }^{2}$ could further complicate our clinical perception. This is an observational study of PU done over a three year period in a multispecialtyhospital. The aim of this study was to do a retrospective analysis of patients with posterior uveitis attending the outpatient department. Analysis was performed to identify frequency of etiology, most common presentation, complication and the cause of visual loss in posterior uveitis. Additionally, we evaluated the clinical response to our treatment protocol which included systemic antibiotics or corticosteroids and immunosuppressive agents.

\section{MATERIALS AND METHODS}

Records of 75 patients who were diagnosed to have PU were studied. This was performed over a period of 3 years and all patients had a minimum follow up for atleast 6 months.

*Corresponding author: Radha Annamalai Ophthalmology, Sri Ramachandra University, Porur
It was done in concurrence with the departments of dermatology, rheumatology and internal medicine. An ethics committee approval was obtained prior to starting the study and patients were enrolled after getting an informed consent. This is a cohort studyand all patients with a clinically proven diagnosis of posterior uveitis were identified and incorporated into the study. An ophthalmic evaluation was performed on all patients. Inclusion criteria were all patients with PU with or without anterior uveitis, vitritis and vasculitis. A detailed history was obtained following which clinicalexamination, slit lamp examination, indirect ophthalmoscopy, biomicroscopy, applanation tonometry and refraction was performed in the out- patient clinic. Ancillary investigations such asfundus photography, fundus fluorescein angiography ${ }^{3}$ was done for all patientsat presentation and subsequently as and when required. Comparisons of the initial and follow up fundus photographs and fluorescein angiograms were made.Optical coherence tomography and b scan ultrasosgraphy were performed in selected cases when indicated for the detection of severity or complications. The clinical features, number of recurrences, final visual acuity and description of healed lesions were documented.

Laboratory tests employed were inclusive of complete blood count, erythrocyte sedimentation rate, purified protein derivative skin test, chest $\mathrm{X}$ ray,VDRL, rheumatoid factor, anti- nuclear antibody and if required QuantiFERON TB Gold $^{4}$ and polymerase chain reaction of intraocular fluid. Specific investigations such as high resolution computed tomography (HRCT), IgG, IgM, lysozyme assay, serum 
angiotensin converting enzyme (ACE), serum calcium, blood culture and enzyme linked immunosorbent assay (ELISA) were performed based on clinical suspicion. Polymerase chain reaction $(\mathrm{PCR})^{5}$ and real time $\mathrm{PCR}^{6}$ was done on aqueous samples.A complete blood count, liver function test, renal function test and blood sugar were done before starting treatment with oral steroids or immunosuppressive agents.

\section{RESULTS}

The median age of presentation was 30-60 years. Recurrences were noted in 12 patients $(16 \%)$ of whom 3 patients had serpiginous choroiditis, 3 patient had tubercular posterior uveitis and 1patients hadtoxoplasma retinochoroiditis.75 patients with a clinical history and features suggestive of posterior uveitis in the form of retinochoroiditis, choroiditis, vasculitis or their complications were studiedfrom 2005 till 2008. Of the 75 patients with posterior uveitis 4 were lost to follow up and excluded from the study.

The break-up of the etiology of the remaining 71 patients was as follows:

Table 1 Etiology of PU Posterior uveitis was bilateral in 43 patients and unilateral in 28 patients.

\begin{tabular}{cc}
\hline Infection: & $\mathbf{2 8}$ patients $(\mathbf{3 7 \%})$ \\
\hline $\begin{array}{c}\text { Autoimmune } \\
\text { diseases: }\end{array}$ & 25 patients $(33 \%)$ \\
Idiopathic: & 18 patients $(24 \%)$. \\
\hline
\end{tabular}

The most common etiology was tubercular posterior uveitis $(48 \%)$ in the form of multifocal serpiginous like choroiditis followed by focal choroiditis, vasculitis and choroidal abscess. The second most common cause of posterior uveitis was toxoplasma retinochoroiditis $(32 \%)$ followed byacute retinal necrosis $(12 \%) .15$ patients had choroiditis and vasculitis and was not attributable to any etiology. The distribution of etiology was as follows

Table 2 Infections: 38(53\%)

\begin{tabular}{cc}
\hline Tubercular uveitis & 17 \\
(TB focal choroiditis & 5 \\
TB multifocal choroiditis & 7 \\
TB vasculitis & 4 \\
TB granuloma ) & 1 \\
Toxoplasma Retinochoroiditis & 5 \\
Acute retinal necrosis & 3 \\
CMV Retinitis & 4 \\
HIV retinopathy & 1 \\
Neuroretinitis & 1 \\
Syphilitis chorioretinopathy & 3 \\
Fungal endophthalmitis & 2 \\
& 3 \\
\hline
\end{tabular}

Table 3 Autoimmune: 28(37\%)

\begin{tabular}{cc}
\hline Serpiginous choroiditis & 11 \\
Peripapillary: & 2 \\
Ampiginous & 9 \\
Vogt- Koyanagi-Harada (VKH) & 4 \\
Acute posterior multifocal placoid pigment epitheliopathy & \\
(APMPPE) & 3 \\
RPE epithelitis & 2 \\
Behcets & 3 \\
Sarcoidosis & 4 \\
PIC & 1
\end{tabular}

In terms ofage, gender and laterality there was no significant difference. Posterior uveitis was seen in 40 males [53\%] and 31 females [41\%]. Anterior uveitis was observed in association with posterior uveitis in $28 \%$ of patients. The most common presentation was choroiditis followed by retinochoroiditis with vitritis. Systemic antibiotics were administered in specific infections. Oral corticosteroids in the form of tablet prednisolone $1 \mathrm{mg} / \mathrm{kg}$ body weight once a day after breakfast with supplements of calcium and antacid were given in isolation or with immunosuppressives ${ }^{7}$. The immunosuppressives used were tablet methotrexate $15 \mathrm{mg}$ per week, tablet azathioprine $150 \mathrm{mg}$ thrice daily for 1 month, $100 \mathrm{mg}$ thrice daily for the $2^{\text {nd }}$ month and $50 \mathrm{mg}$ as maintenancedose for the next 2 months and tapered depending on the response of the patient. In patients who were intolerant to steroids, immunosuppressive therapy were added or substituted if there was no response or worsening after $1-2$ weeks. Blood counts, liver function and kidney function tests were monitored in these patients. The visual outcome in these patients was assessed and graded as significant improvement (more than 3 lines), improvement (more than 2 lines), no improvement and deterioration. Significant improvement of vision was seen in 15 patients $(2 \%)$, marginal improvement in 37 patients $(45 \%)$, vision remained the same in 7 patients (1\%) and deterioration in 13 patients (17\%). Tubercular posterior uveitis had the best visual outcome following treatment and the bestprognosis as seen in 9 patients with improved acuity. Macular involvement in our series was seen in 28 patients [37\%] and the etiology in these patients was VKH, macular serpiginous choroiditis, APMPPE, neuroretinitis, retinal pigment epithelitis and toxoplsma retinochoroiditis. Patients with VKH with macular involvement were admitted and administered IV methyl prednisolone in the dose of $1 \mathrm{gm}$ daily for 3 days followed by tablet prednisolone I $\mathrm{mg} / \mathrm{kg}$ body weight. Improvement of vision occurred in VKH. However 7 patients withserpiginous choroiditis had a drop in vision even following treatment.

Table 4 Macular involvement

\begin{tabular}{cl}
\hline Serpiginous choroiditis & $\mathbf{9}$ \\
\hline VKH & 5 \\
Toxoplasma retinochoroiditis & 6 \\
Neuroretinitis & 4 \\
APMPPE & 3 \\
Retinal pigment epithelitis & 1 \\
\hline
\end{tabular}

Complications were seen in 24 patients amounting to $32 \%$. Macular scarring in 7patients and cystoid macular scarring in 7 patients were seen. Choroidal neovascular membranes (CNVM) in3, tractional retinal detachment in 1 and vitreous haemorrhage in 1 patient was observed. Macular scarring developed in 3 patients with serpiginous like tubercular posterior uveitis, 2 patients with ampiginous choroiditis, 2 patients with toxoplasmic retinochoroiditis. Cystoid macular oedema was seen in 4 patients with VKH syndrome and 2 patients with neuroretinitis. CNVM was seen in 2 patients with macular serpiginous choroiditis and 1 with toxoplasma retinochoroiditis. Tractional bands and epiretinal membranes were seen in patientswith Eales disease. In autoimuune posterior uveitis macular scarring and choroidal neovascular membranes were seen most frequently as complications.

\section{DISCUSSION}

Posterioruveitis could be due to an infection, autoimmune disease process or simply idiopathic. Investigations that are done may not be sufficient and ancillary testing with laboratory testing using intraocular fluid assay following anterior chamber tap if required. In our study during analysis 
of posterior uveitis which had been diagnosed as idiopathic we found 3 patients who had evidence of latent tuberculosis by QuantiFERON TB Gold test ${ }^{8}$ when investigated further and they were subsequently treated with 4 drug regimen of anti- tuberculosis therapy (ATT). They had no evidence of systemic TB and were negative for X-ray chest and Mantoux test.

In our study, posterior uveitis was most commonly due to TB. Age groups affected ranged from 19 to 70 years thus proving that choroidal involvement in TB was seen across all age groups and was due to multiple types of systemic TB such as underlying lung $\mathrm{TB}$, central nervous system $\mathrm{TB}$ in varied forms, abdominal TB or miliary TB. However it was not frequently seen in active systemic TB. Several patients had latent TB with no manifestation of the disease which was detected with quantiFERON TB Gold. The diagnosis of PU due to TB in the absence of systemic TB is difficult as sometimes even microbiological analysis of intraocular fluids is negative. This may be because the bacilli reside in the retinal pigment epithelium (RPE) and the inflammation itself could be due to a hypersensitivity reaction to the tubercle bacillus and not always due to active infection by the organism 9 . On analysis inour patients with tubercular PU, serpiginous- like choroiditis followed by multifocal choroiditis (Fig. 1) was the most common manifestation and is believed to occur due tohypersensitivity to the DNA of the TB bacillus. These lesions resolved with a nine month course of ATT and steroids.

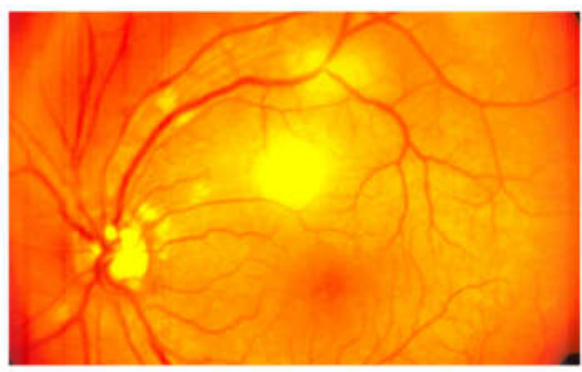

Figure 1 Mulrifocal choroiditis due to TB in the left eye

A few of our patients had subretinal abscess which was due to necrosis and liquefaction which occurs within a tuberculoma. There was associated vitritis and vasculitis. Another frequent manifestation was Eale's disease. Choroidal tubercles in our study was seen in patients with military TB. They had no associated vitritis but surrounding subretinal fluid was noted. $\mathrm{B}$ scan needs to be performed in patients with choroidal tuberculoma to differentiate this from a tumour. Besides the variable presentation of multifocal tubercular posterior uveitis can resemble ampiginous choroiditis ${ }^{9}$ and thus further complicate the scenario. This similarity in presentation has been reported by Gupta et $a l^{10}$ as presumed tubercular serpiginous choroiditis ${ }^{11}$. In our study three patients had presented with tubercular serpiginous like choroiditis and one of the three had been subjected to AC Tap and the aqueous humor analysed using PCR. The usefulness of AC tap has been studied and reported by Rothova et $a l^{12}$. The other two patients had been sent for QuantiFERON TB Gold testing and latent tuberculosis was detected. Realtime $\mathrm{PCR}^{13}$ can also be employed to analyse the aqueous humor sample to detect infection and it has the advantage of providing evidence and quantification of the microbe. Tubercular $\mathrm{PU}^{14}$ is best diagnosed based on a combination of clinical signs and by demonstration of bacilli on culture or DNA amplification using PCR or RT- PCR. When this is not possible, a presumptive diagnosis can be arrived at using frequently noted signs with tests such as Mantoux test ${ }^{15}, \mathrm{HRCT}^{16}$, quantiFERON TB or based on response to treatment. The inferences that we drew with regard to individual entities studied are as follows:

TB choroiditis and vasculitis followed by toxoplasma retinochoroiditis was the most frequent form of posterior uveitis due to infectious etiology. All our patients were immunocompetent and belonged to $4^{\text {th }}$ to $5^{\text {th }}$ decades of life. A positive history of contact with pets, ingestion of undercooked meat or water contamination was present. We observed that congenital toxoplasmosis was less frequent than acquired toxoplasmosis. It was unilateral in all our patients and was seen as focal necrotizing retinitis at the macula with profuse vitritis. Vasculitis around the lesion was seen in the active stage of retinitis due to antigen- antibody reaction or deposition along the venules. Multifocal involvement though described was not seen in our patients and all lesions were seen adjacent to scars suggestive of reactivation. None of our patients had anterior segment involvement or secondary glaucoma.

PU due to virus in the form of acute retinal necrosis was seen in few of our patients. The classic triad of arteritis, retinitis and vitritis with peripheral involvement and rapid spread was noted. All patients responded to high dose intravenous acyclovir and had good visual recovery with no complications. HIV retinopathy was diagnosed in two patients who presented with cotton wool spots and otherwise normal fundus. Both the granular and fulminant form of CMV retinitis was seen in our patients. Candida retinochoroiditis occurred in three patients all of whom were diabetics. Blood cultures were negative and a diagnosis was made based on intraocular fluid assay using microbiological culture. The patient was successfully treated using $1 \mathrm{mg} / \mathrm{kg}$ per day of intravenous amphotericin B for 2 weeks. After the lesion showed signs of resolution, it was changed to oral fluconazole, $200 \mathrm{mg}$ twice daily following which visual improvement was noted.

Serpiginous choroiditis (Fig. 2) was the most common cause in the autoimmune group in our patients. We saw all three types of morphological presentations which included peripapillary, macular or ampiginous types.

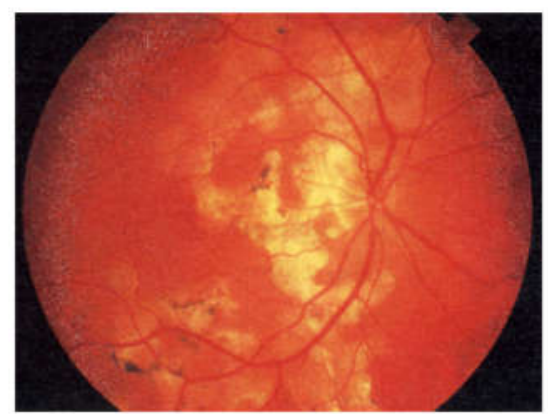

Figure 2 Serpiginous choroiditis in the right eye

Acute retinal pigment epithelitis (ARPE- Krill's disease) was seen in many patients and was found to be a more frequent cause of PU than described in literature. It was typically unilateral with macular lesions and subtle changes in the RPE and was self-limiting with a good prognosis. 
In $\mathrm{VKH}$ syndrome this disease was seen to have bilateral presentation with multifocal exudative detachment (Fig. 3) and panuveitis with or without disc oedema. The majority of patients whom we saw had the probable type of VKH where neurological and integumentary signs were absent. FFA showed patchy hyperfluorescence with pinpoint leaks at the level of RPE which was confirmed by OCT. Treatment in the form of oral steroids has to be continued for atleast 6 months in order to prevent recurrence.
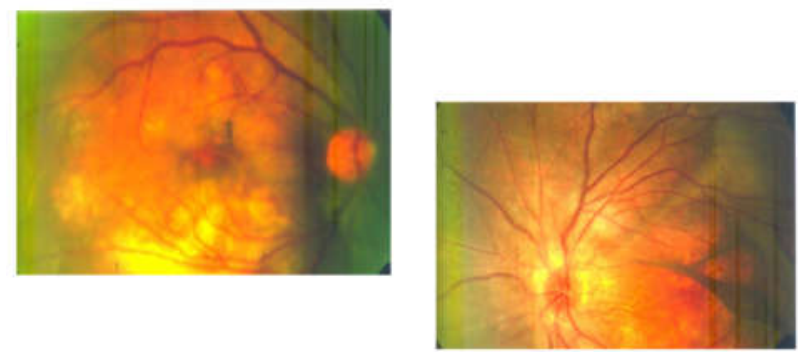

Figure 3 Bilateral serous detachment with macular involvement in VKH syndrome

Sarcoidosis was seen in both younger and elderly age groups. Most patients had choroidal granulomas or acute anterior uveitis. Patients need immunosuppressive therapy and treatment was done in concurrence with the physician.

The commonest cause of vasculitis that we encountered was due to Eale's disease. All patients were young and had unilateral vitreous haemorrhage at presentation. Examination of the fundus of the other eye showed peripheral vasculitis. A work up for tubercular etiology wass done in these patients and treatment with ATT and/or steroids was started. Complications such as neovascularisation elsewhere or on the disc can develop because of non- perfusion and ischemia. This may require laser photocoagulation to prevent vitreous haemorrhage.

In our population where infections are abundant we inferred that posterior uveitis was most commonly due to systemic infections. The onset of signs in these patients does not have to respect any set criteria and variations in clinical presentation were observed even amongst the same infection.

The involvement of the macula with visual loss occurred most commonly with autoimmune diseases such as VKH and macular serpiginous choroidopathy. Among infections it was only toxoplasmosis which predominantly involved the macula. Tubercular choroiditis caused extensive lesions at the posterior pole but did not affect the macula directly in most patients. Even when the macula was affected, the macular lesions resolved completely with ATT and did not contribute to vision loss.

The most common presentation was choroiditis followed by retinochoroiditis with vitritis. Scarring and choroidal neovascular membranes were noted as the most frequent cause of defective vision in many patients. In those with CNVM, FFA was performed to identify the type and location of the membrane. They were treated with laser photocoagulation or anti- VEGF agents and visual recovery in most was good. However, recurrence of membranes was a significant complication in our patients.

\section{CONCLUSIONS}

Posterior uveitis in our setting was observed in the $4^{\text {th }}$ decade of life and was mostly due to infection that predominantly presented with choroiditis. However the variations that we observedwas that some of our patients who were considered idiopathic had an underlying systemic infection which had not yet fully evolved and remained masked but when more precise investigations were employed their evidence was identified. This has led us to believe that infections may present with posterior uveitis before becoming apparent as systemic disease. Characteristic clinical features maynot always be present in $\mathrm{PU}^{17}$. Differentiation between infections and autoimmune type is very important as treatment is totally different. Infections need to be treated with specific antimicrobials such as $\mathrm{ATT}^{18,19}$ and steroids. Emperical treatment with corticosteroids and immunosuppressives should not be given in all patients with PU. Ancillary tests ${ }^{20}$ and aqueous humor analysis ${ }^{21}$ helps in establishing the diagnosis. Follow up is important to look for recurrence and complications because early diagnosis and correct treatment can prevent visual loss.

\section{References}

1. Majumder PD, Ghosh A, Biswas J. Infectious uveitis: An enigma. Middle East Afr J Ophthalmol. 2017; 24(1):2-10.

2. D G Charteris, W R LeeRamasamy Krishnadas, Rengapa Ramakrishnan,Ravilla D Thulasiraj, James M Tielsch, Joanne Katz, Alan L Robin, H Kempen, Multifocal posterior uveitis: clinical and pathological findings. $\mathrm{Br} J$ Ophthalmol 1990; 74;688-693

3. Current approach in the diagnosis and the management of posterior uveitis. Sudha K Ganesh, Jyotirmay Biswas. Indian Journal of Ophthalmology, 2009; 58 (1): 29-43.

4. Kalpana Babu et al Utility of QuantiFERON Gold in a South Indian population with ocular inflammation Indian Journal of Ophthalmology. 2009; 57 (6): $427-$ 430.

5. Application of PCR to ophthalmic diseases. Survey of Ophthalmology, November2001:Volume46, Issue 3, Pages 248-258.

6. Santos FF, Commodaro AG, Souza AV, Pinho JR, Sitnik R, Garcia C, Ribeiro AL, Muccioli C, Lottenberg CL, Rizzo LV, Belfort Junior R. Realtime PCR in infectious uveitis as an alternative diagnosis. Arq Bras Oftalmol. 2011; 74(4):258-61.

7. Douglas A. Jabs, James T. Rosenbaum, C.Stephen Foster, Gary N. Holand, et al. Guidelines for the use of immunosuppressive drugs in patients with ocular inflammatory disorders: recommendations of an expert panel October 2000 American Journal of Ophthalmology Vol. 130, Issue 4, Pages 492-51

8. Mackensen, Matthia D, Bcker, Ute Wiehler, Regina,, Max. Alexander DalpkeStefan Zimmermen QuantiFERON TB-Gold-A New Test Strengthening Long-Suspected Tuberculous Involvement in Serpiginous-like Choroiditis American Journal of Ophthalmology. 2008; 146 (5): 761-766.

9. Rao NA, Saraswathy S, Smith RE. Tuberculous uveitis: Distribution of Mycobacterium tuberculosis in 
the retinal pigment epithelium. Arch Ophthalmol 2006; 124:1777-9.

10. Gupta V, Gupta A, Arora S, Bambery P, Dogra MR, Agarwal A. Presumed tubercular serpiginouslike choroiditis: Clinical presentations and management. Ophthalmology 2003; 110:1744-9

11. Gupta V, Gupta A, Rao NA. Intraocular tuberculosisan update. Surv Ophthalmol 2007; 52(6):561-87

12. Rothova A, de Boer JH, Ten Dam-van Loon NH, Postma G, de Visser L, Zuurveen SJ, Schuller M, Weersink AJ, van Loon AM, de Groot-Mijnes JD. 15. Usefulness of aqueous humor analysis for the diagnosis of posterior uveitis. Ophthalmology. 2008;115(2):306-11

13. Dworkin LL, Gibler TM, Van Gelder RN. Real time Quantitative Polymerase chain Reaction Diagnosis of Infectious Posterior Uveitis, Arch Ophthlmol. 2002; 120 (11): 1534-9.

14. Amod Gupta, Reema Bansal, Vishali Gupta, Aman Sharma, Pradeep Bambery Ocular Signs Predictive of Tubercular Uveitis. Am J Ophthalmol 2010; 149 (4): 562- 570 .
15. Jyotirmay Biswas Practical concepts in the management of uveitis Ophthalmology. Indian Journal of Ophthalmology Year 1993; 41(3): 133-141

16. Ganesh $\mathrm{SK}^{1}$, Roopleen, Biswas J, Veena N. Role of high-resolution computerized tomography (HRCT) of the chest in granulomatous uveitis: a tertiary uveitis clinic experience from India. Ocul Immunol Inflamm. 2011; 19(1):51-7.

17. SR Rathinam, P Namperumalsamy Global variation and pattern changes in epidemiology of uveitis. Indian Journal of Ophthalmology. 2007; 55(3): 173-183.

18. Abrams J, Schlaegel TF Jr. The role of the isoniazid therapeutic test in tuberculous uveitis. $\mathrm{Am} J$ Ophthalmol 1982; 94:511-5

19. Centers for Disease Control and Prevention. Treatment of Tuberculosis. MMWR Recomm Rep. 2003; 20; 52(RR-11):1-77.

20. Carl P Herbort. Fluorescein and Indocyanine Green Angiography for Uveitis. Middle East Afr $J$ Ophthalmol. 2009; 16(4): 168-187.

21. Chronopoulos A, Roquelaure D, Souteyrand G, Seebach JD, Schutz JS, Thumann G. Aqueous humor polymerase chain reaction in uveitis - utility and safety. BMC Ophthalmol. 2016 .28; 16(1):189.

\section{How to cite this article:}

Radha Annamalai et al (2017) ' A Clinical Profile Of Posterior Uveitis In A Multispecialty Hospital', International Journal of Current Advanced Research, 06(05), pp. 3943-3947.

DOI: http://dx.doi.org/10.24327/ijcar.2017.3947.0402 